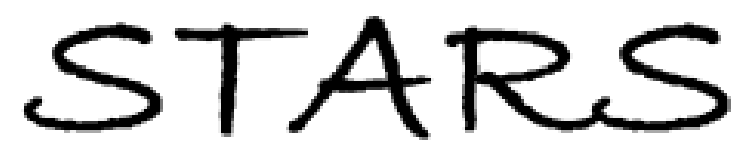

University of Central Florida

STARS

$1-1-2000$

\title{
Duration and exposure to virtual environments: Sickness curves during and across sessions
}

\author{
Robert S. Kennedy \\ Kay M. Stanney \\ University of Central Florida \\ William P. Dunlap
}

Find similar works at: https://stars.library.ucf.edu/facultybib2000 University of Central Florida Libraries http://library.ucf.edu

This Article is brought to you for free and open access by the Faculty Bibliography at STARS. It has been accepted for inclusion in Faculty Bibliography 2000s by an authorized administrator of STARS. For more information, please contactSTARS@ucf.edu.

\section{Recommended Citation}

Kennedy, Robert S.; Stanney, Kay M.; and Dunlap, William P., "Duration and exposure to virtual environments: Sickness curves during and across sessions" (2000). Faculty Bibliography 2000s. 2641. https://stars.library.ucf.edu/facultybib2000/2641

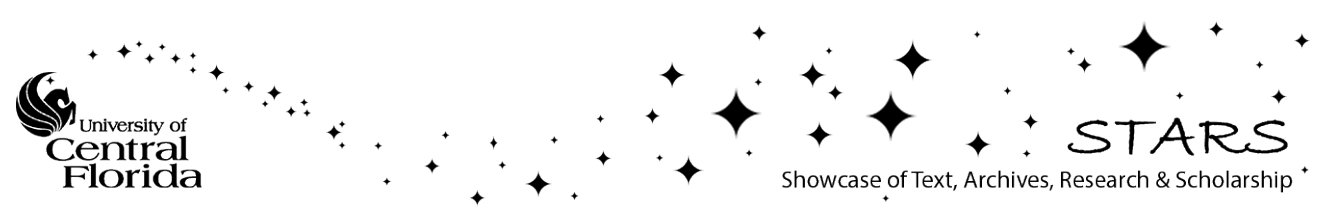




\section{Robert S. Kennedy}

RSK A ssessments

1040 W oodcock Rd, Suite 227

0 rlando, FL 32803

\section{Kay M. Stanney}

University of Central Florida

D epartment of Industrial Engineering

and Management Systems

0 rlando, FL 32816

\section{William P. Dunlap}

Tulane U niversity

Department of Psychology

N ew 0 rleans, LA 70118
Presence, Vol. 9, N 0. 5, October 2000, 463-472 (1) 2000 by the Massachusetts Institute of Technology

\section{Duration and Exposure to Virtual Environments: Sickness Curves During and A cross Sessions}

\section{Introduction}

There is definite concern that simulator sickness could prevent virtual environment (VE) technology from realizing its full potential. Considerable research has been focused on motion and simulator sickness, but with no satisfactory solution resulting. As Biocca stated, simulator sickness may be "a 'snake' that will linger (indefinitely) in the underbrush of virtual worlds"' (Biocca, 1992, p. 341). Whether or not the symptomatology and causative drivers of motion sickness can be completely specified, it is essential to identify a means to control the deleterious effects of illness resulting from VE exposure.

The symptomatology of motion sickness generally manifests itself in three ways: an emetic response, oculomotor disturbances such as eyestrain and/ or disorientation; ataxia (postural instability); and vertigo (Kennedy, Berbaum, \& D rexler, 1994). V isually interactive systems, such as virtual environments, generally produce more oculomotor-related symptoms and ataxia than actual vomiting does (Kennedy et al., 1992; Kennedy \& Lilienthal, 1995). With this knowledge, one can reasonably predict the types of aftereffects that users of $V E$ systems will experience.

The cau sative drivers of motion sickness that are generally explored include those related to technical system factors and user characteristics. M any re- 
searchers support the view that simulator sickness is a technical problem that can be eliminated through technological advances. Considerable investigation has focused on the technical factors suggested to cause simulator sickness (Biocca, 1992; Kolasinski, 1995; Pausch, C rea, \& Conway, 1992). These factors include positiontracking errors, optical distortion, field of view, scene content, flicker, motion platforms, refresh rate, resolution, transport delays, and update rate. 0 thers believe that motion-sickness susceptibility is driven by individual differences among users, and thus can probably never be completely resolved. Factors such as user experience, gender, field independence, age, illness, flicker fusion frequency threshold, mental rotation ability, postural stability, and susceptibility to vection have all been shown to relate to sickness susceptibility in one way or another. (See Kolasinski (1995) for a comprehensive review of technical and user factors affecting simulator sickness.) H ettinger et al. (1990) demonstrated that vection in a simulator was a key element in the development of sickness symptoms. Vection is the highly compeling perception of self-motion in physically stationary observers that may be achieved by highly realistic or wide fieldof-view displays of optical flow patterns ( $\mathrm{H}$ ettinger \& Riccio, 1992). This suggests that technological advances that improve the realism and compeling nature of simulations may actually increase rather than decrease the incidence and severity of sickness. It should be emphasized, however, that individuals who do not experience vection generally do not develop motion sickness symptoms (H ettinger et al., 1990).

Theoretical treatments of these system and user factors, aimed at synthesizing and summarizing the literature, have failed to produce a general predictive model of cybersickness, although there are models of motion sickness in general (Crampton, 1990). The lack of a predictive $V E$ sickness model may be the result of the fact that these technical and user factors, even when considered together, do not account for as much of the variance in sickness outcome as two prevailing obvious factors. The temporal aspects of exposure (i.e., exposure duration and time between exposures) are factors that have been largely neglected from systematic evaluations. We think $20 \%$ to $50 \%$ is a conservative estimate of the amount of variance in motion-sickness outcomes (i.e., incidence and severity) due to these temporal factors. Temporal aspects have two main components: 1) the amount of time that a person is exposed to a VE, and 2) the distribution of exposures over time (viz., days). Each of these can be expected to have a significant effect on the severity of sickness experienced.

Although all effects generally interact, the effects of time on cybersickness may be largely additive (or subtractive if exposure time is reduced!). If this is true and the effects are sizable, three implications should be recognized. First, the temporal effects in any cybersickness experiment should be covaried or controlled in order to study experimentally the other technical and user-related effects mentioned above. Second, from a practical standpoint, this information could be used to control sickness outcomes by manipulating exposure duration. Third, it will not be possible to perform quantitative meta-anaIytic comparisons of the variance accounted for by the disparate determiners of sickness unless time is taken into account.

\subsection{Exposure Duration}

It is well known that exposure duration can influence sickness rates, and most reviewers mention the importance of exposure duration and repeated exposures; yet limited systematic research has been reported in this area. M iller and Graybiel (1970a) provided early evidence that the longer the exposure time, the greater the sickness outcome experienced by individuals. Lampton et al. (1994) found, in general, that the longer the exposure duration to a VE, the more severe the resulting sickness symptoms over a $40 \mathrm{~min}$. exposure period. Similarly, R egan (1995) reported that subjects experienced progressively more sickness symptoms as exposure duration in a VE continued over a 20 min. period. Anecdotal evidence from M cC auley and Sharkey's research led them to suggest that "exposure time should be limited until adaptation to the VE has occurred" (M cC auley \& Sharkey, 1992, p. 316). This suggestion was based on the fact that, in their research, the probability of experiencing negative aftereffects from VE exposure increased as the exposure time increased. Guignard and M cC auley 
(1982) found that the time course of sickness-incidence level increases steadily for the first $60 \mathrm{~min}$. of exposure, at which point it reaches a threshold level of approximately $80 \%$

These studies indicate that, in general, when exposure is continued, one finds that the longer a person is exposed, the greater the sickness outcome. Indeed, this is a central rationale behind a well-known provocative test of motion sickness susceptibility known as the Coriolis Sickness Susceptibility I ndex (CSSI) (M iller \& Graybiel, 1970b). The C SSI requires subjects to perform head movements while rotating at a constant velocity in a servo-controlled chair. In this test, subjects are continuously challenged by increments in rotational velocity and time cojointly, and a subject's CSSI score is a combination of duration and intensity.

Thus, while length of exposure has an obvious relationship to the incidence of sickness (which is assumed by nearly every writer on the topic), there are inexplicably almost no quantitative data relating length of exposure to motion sickness. The only known predictive model that takes exposure duration into consideration is the M otion Sickness D ose Value (M SD V) which is based on seasickness data (British Standards I nstitute, 1986; Griffin, 1986). This model provides a theoretically quantitative prediction of the level of expected motionsickness incidence (M SI) based on exposure time and the acceleration magnitude and frequency of passive motion. T wo issues limit this model's generalizability. First, the model has yet to be fully validated. As G uignard and M cCauley (1990) note, "the M SD V still appears to be an elegant edifice of hypothesis based as yet upon a somewhat sketchy base of hard data relating M SI to measured inertial inputs." Secondly, this model is based on the M SI experienced during passive whole-body oscillation (that is, ship heave motion), which does not parallel the virtual motion experienced in visually interactive environments. There is thus a need to develop a predictive model that relates specifically to virtual environments.

The issue is to determine the form of the relationship between temporal factors and sickness outcome experienced in visually interactive systems and whether this form is generalizable across different system conditions.
The few existing studies with large sample sizes in which sufficient information was provided in the various reports to determine the effect of duration on illness are as follows.

- In a simulated sea-sickness experiment in which a ten-point rating scale of severity was used (Guignard \& M cCauley, 1982), there was a linear trend in sickness ratings over the course of the experiment.

- Data from a large $(N>1000)$ simulator survey (Kennedy \& Fowlkes, 1990), showed a correlation $(r=0.50, p<0.05)$ between average sickness and length of exposure in sixteen different simulators.

- A recent VE study (Lampton et al., 1994) reported a linear trend in three experiments using different protocols.

In these studies, although duration was not a central issue in any experiment, sufficient information was available to evaluate that a positive relationship existed.

Taken together, these results confirm the logical and expected correspondence between sickness and exposure duration. Longer exposures produce more symptoms. 0 ther than that, the relationship is likely to be mainly monotonic. The precise form of this relationship is not known, nor whether the same form can be expected in all environments. Because of the potential influence of this relationship in any examination of simulator sickness or related human-factors problems, it is surprising that, when comparisons among devices are performed, the evaluators do not explicitly take into account the length of the exposure under conditions where sickness or other aftereffects are found. Therefore, it is suggested that any systems or procedural specifications developed for managing simulator sickness should include the duration of time over which the individual is exposed.

\subsection{Repeated Exposures}

O ne of the most pervasive characteristics of the nervous system is that it is adaptive. With respect to motion sickness, adaptation is almost al ways reported when people are exposed repeatedly. D ue to adaptation, response reduction is the normal consequence of such repetitive stimulation. The result of this adaptation is that 
people who work with VR devices and flight simulators will likely become habituated to the devices they use routinely. This can be expected to occur with most systems, and thus there is likely to be a tendency, based on their own current experience, that systems developers or administrators will underestimate the severity and incidence of aftereffects from exposure. Thus, while developers understand that cybersickness is reduced over repeated sessions, it would be useful to determine the shape of this illness-time function. Such information could be used to manage the deleterious aftereffects of VE exposure.

Reason (1970) found that $95 \%$ of normal individuals demonstrate significant adaptation to the conditions that produce motion sickness, once given prolonged exposure to a provocative stimulus. Although this adaptation can occur with prolonged exposures, it appears to occur more effectively and efficiently with repeated exposures. An exception is the increased adaptation with massed exposure using a titrated stepwise increase in velocity in the Pensacola Slow R otation R oom (Reason \& Graybiel, 1970).

Limited data are available to characterize the adaptation of cybersickness with repeated exposures. Biocca (1992) cites evidence that the occurrence and severity of sickness noticeably decreases after four to six exposures in simulators. M CC auley and Sharkey (1992) found that adaptation was facilitated by subjecting users to brief repeated exposures. Gower et al. (1987) and Kennedy et al. (1993) found reduced sickness in flight simulators over successive flights. R egan (1995) found a dramatic decrease in sickness incidence over the course of four repeated exposures, and Kennedy et al. (1996) found marked reductions in sickness in the second of two 40 min. VE exposures. The results of the Kennedy et al. (1993) study suggested that, for repeated exposures to be effective in desensitizing individuals to sickness, the intersession interval should be short (one week or less). This finding is consistent with reports from other forms of motion sickness in which seven-day intervals between Slow Rotation Room exposures provided savings but thirty days did not (Kennedy, Tolhurst, \& Graybiel, 1965), and in which intersession intervals of one day or less showed no evidence of increased tolerance, nor did those greater than six days apart, while those two to five days apart appeared optimum (Kennedy et al., 1993).

\subsection{Study Objectives}

These results taken together indicate that sickness incidence can be expected to increase monotonically over time, until such time that end points (such as vomiting, retching, collapse, shock, and sleep) are reached, or adaptation ensues. Further, adaptation can generally be facilitated through repeated, brief exposures. The objective of the current study was to evaluate the generalizability of the relationships between sickness, exposure duration, and repeated exposures over diverse systems and large subject pools. If these functions are found to be generalizable, they could be used as prescriptive tools to minimize the incidence and severity of sickness related to human-virtual environment interaction.

\section{Method}

T wo evaluations were performed to characterize the function of sickness outcomes. The first examined exposure duration within a session, and the second evaluated repeated exposures across sessions. In both evaluations, the total sickness scores were based on the Simulator Sickness Q uestionnaire (SSQ) ( L ane \& Kennedy, 1988). The SSQ consists of a checklist of 26 symptoms, each of which is related in terms of degree of severity (none, slight, moderate, severe), with the highest possible total score being 300. A weighted scoring procedure is used to obtain a global score reflecting the overall discomfort level known as the Total Severity (TS) score. The SSQ also provides scores on three subscales that represent separable, but correlated, dimensions of simulator sickness (nausea, visuomotor disturbances, and disorientation). Because the total score is a composite of the three factor scores, it can be expected to be the most reliable, and TS was used in this analysis.

\subsection{Exposure Duration}

To assemble sufficient data for this analysis, 938 cases of exposure duration in a variety of simulators and 
scenarios were reexamined. These cases were extracted from a large database of flight simulator data which has been collected over the past eleven years for various military agencies. For this study, only helicopter flights were analyzed, because fixed-wing simulators tend to be less provocative than helicopter simulators (Kennedy et al., 1993)

The simulator flights were divided into categories based on duration of exposure, because varying exposure duration times were used in the different experimental data sets evaluated. Four categories of duration times were studied: 0 to $1 \mathrm{hr}$.; 1 to $2 \mathrm{hr}$;; 2 to $3 \mathrm{hr}$;; and 3 or more hours. M ost of the simulated flights examined were between 1 and $2 \mathrm{hr}$. in duration. Flights of more than $3 \mathrm{hr}$. were considerably less common. The total motion-sickness scores of each category were analyzed using a one-way analysis of variance (AN OVA). The a priori prediction was that a significant positive linear trend would result relative to the duration categories.

\subsection{Repeated Exposures}

In contrast to the preceding analysis, the data set used for the evaluation across sessions came from a single simulator from which a large amount of trainees' data was available. The simulator was a training helicopter (TH -57C) at the $\mathrm{N}$ aval Air Station (N AS) Whiting, in M ilton, Florida. A total of 53 complete records of sickness scores across seven consecutive flights were isolated for study. These data were analyzed with a repeated-measures AN OVA, where flight number was a within-subjects variable and total sickness score was the dependent variable. It was predicted that a meaningful change in sickness, such as that produced by adaptation, would demonstrate a significant negative linear trend with respect to flight number. Sickness was thus expected to decrease with trials.

\section{$3 \quad$ Results}

\subsection{Exposure Duration}

D escriptive statistics for the exposure duration across duration categories are provided in Table 1. Figure 1 depicts the means and standard-error bars as a
Table 1. Descriptive Statistics for Total Sickness Reported after Various Durations of Stimulation in Helicopter Flight Simulators

\begin{tabular}{lllll}
\hline \multicolumn{5}{c}{ D uration (hours) } \\
\cline { 2 - 5 } & $0-1$ & $1-2$ & $2-3$ & 3 or more \\
\hline M ean & 10.78 & 14.09 & 14.67 & 17.48 \\
S.D. & 15.42 & 14.62 & 18.55 & 17.29 \\
N & 85 & 672 & 129 & 52 \\
\hline
\end{tabular}

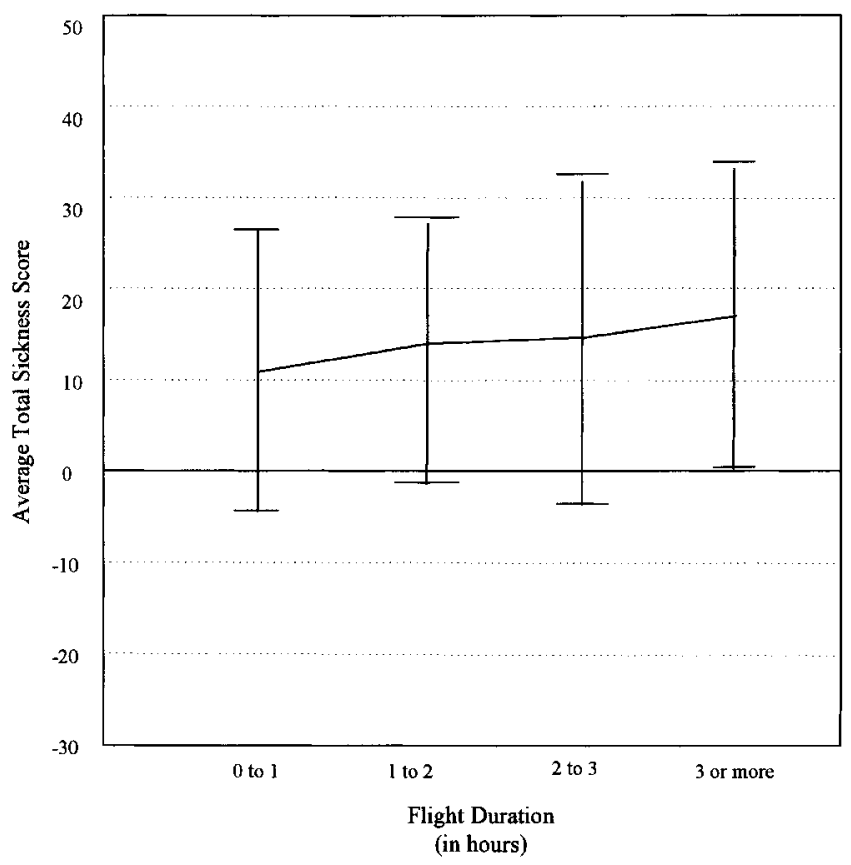

Figure 1. M ean total reported sickness score, with standard error bars, as a function of flight duration in a variety of helicopter flight simulators.

function of flight-duration category. As expected, the means monotonically increase with respect to flight duration. Analysis of variance results showed a significant linear trend $(F(1,934)=5.36, p=0.02)$ corresponding to the monotonic changes in sickness score (see Table 2). $\mathrm{H}$ igher-order trend components were not significant. Although the sample size was large, it should be noted that a number of different simulators were represented, and the scenarios also varied within simulators. Therefore, there was considerable opportunity for variance of 
Table 2. Anova Table for Total Sickness Reported after Various Durations of Stimulation in Helicopter Flight Simulators

\begin{tabular}{|c|c|c|c|c|c|}
\hline Source & $\begin{array}{l}\text { Sum of } \\
\text { squares }\end{array}$ & d.f. & $\begin{array}{l}\text { M ean } \\
\text { square }\end{array}$ & $\mathrm{F}$ & $\begin{array}{l}\mathrm{p} \text { - } \\
\text { value }\end{array}$ \\
\hline \multicolumn{6}{|l|}{ Duration } \\
\hline Category & 1570.17 & 3 & 523.39 & 2.20 & 0.09 \\
\hline Linearity & 1277.57 & 1 & 1277.57 & 5.36 & 0.02 \\
\hline \multicolumn{6}{|l|}{$\begin{array}{l}\text { Deviation } \\
\text { from }\end{array}$} \\
\hline Linearity & 292.60 & 2 & 146.30 & 0.61 & 0.54 \\
\hline Error & 222679.94 & 934 & 238.42 & & \\
\hline Total & 224250.11 & 937 & & & \\
\hline
\end{tabular}

Table 3. Descriptive Statistics for Total Sickness Reported across Seven Consecutive Flights in a Training Helicopter (TH -57C at N AS W hiting)

\begin{tabular}{lrrr}
\hline Flight number & M ean & Standard deviation & N \\
\hline 1 & 15.53 & 22.03 & 53 \\
2 & 10.73 & 30.12 & 53 \\
3 & 4.38 & 7.29 & 53 \\
4 & 4.31 & 7.53 & 53 \\
5 & 2.40 & 5.03 & 53 \\
6 & 2.26 & 4.94 & 53 \\
7 & 1.55 & 4.11 & 53 \\
O verall & 5.88 & 15.63 & 371 \\
\hline
\end{tabular}

other sources to occur. It is thus not surprising that the $F$ value was not larger.

\subsection{Repeated Exposures}

$M$ eans and standard errors for the repeated exposures data are presented in Table 3 and Figure 2. As can be seen in the figure, there was a monotonic decrease in sickness as a function of flight number. In addition, owing to the minimum score of zero sickness, there was a floor effect causing deceleration in the decline of sickness. The drop in sickness with repeated flights was supported by a significant linear trend $(F(1,52)=17.06$,

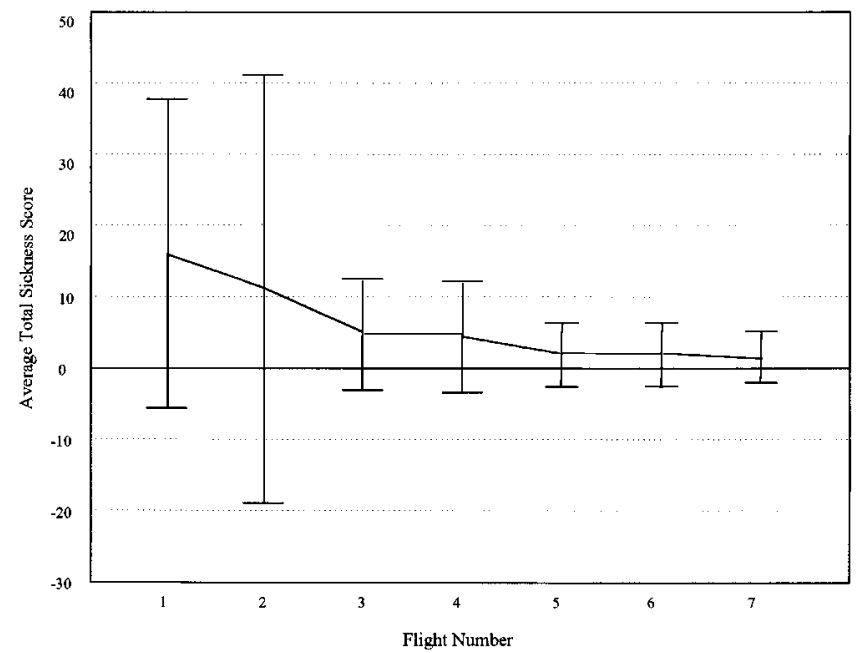

Figure 2. M ean total reported sickness score, with standard error bars, as a function of number of successive flights in a single helicopter flight simulator.

$p=0.0001)$, and the curvature of the function showed a significant quadratic component $(F(1,52)=11.21$, $p=0.0015)$. No other higher-order trend component was statistically significant.

\section{Discussion}

The results indicate that exposure duration and repeated exposures are linearly related to sickness outcomes. As expected, as the length of the exposure increased, the total sickness reported also increased. Although this finding is encouraging, additional study is advised. In examining the mean score of the 0 -to- 1 duration category $(\bar{X}=10.78$, s.d. $=15.42)$, it was determined that, based on the results of another study (Kennedy, Stanney, \& Jones, 1996), this group is at the 75th percentile of typical sickness outcomes. Thus, if the duration-exposure function was more definitively characterized between 0 and $1 \mathrm{hr}$., a "safe" exposure duration may be identified that could drop the total sickness level reported to below the 35th percentile, a zero-point (no sickness symptoms) for military pilots (see Figure 3 ) as determined by Kennedy et al. (1996). It is important to note that the zero-point for the general population of VE users may be significantly lower. Kolasinski (personal 


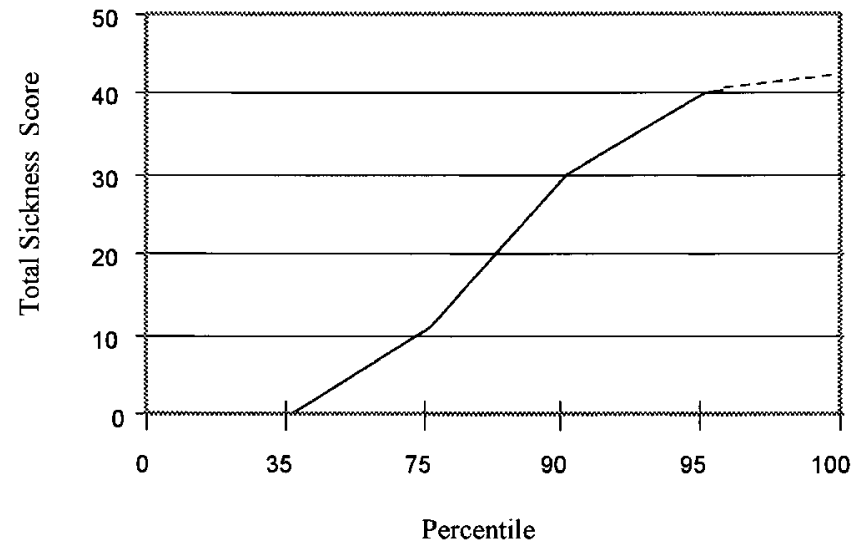

Figure 3. Percentile data for total sickness scores.

communication, 1996) found a zero-point at approximately the 5 th percentile in a recent VE study. The implication is that this function can help to identify exposure-duration times that minimize the negative effects of human-VE interaction.

The significant negative linear trend with respect to repeated flights indicates that M cC auley and Sharkey's (1992) suggestion to use short, repeated exposures to manage simulator sickness may be an effective strategy. The questions remaining to effectively implement such a strategy are:

1. H ow short is a "short" duration? (That is, the duration curve must be better defined between 0 and $1 \mathrm{hr}$.)

2. What is the optimal intersession interval to facilitate adaptation?

3. Can duration exposure increase with repeated exposures?

4. While sickness may be managed by such techniques, do other aftereffects occur such as disturbance in balance or felt limb position? And, if so, how should this be managed?

Although considerable study is needed to realize the answers to these questions, the results could be used to significantly reduce the negative effects of VE exposure.

Beyond modifying experimental or practical-use protocols, data on the linear nature of simulator sickness over prolonged or repeated exposures could be used to derive or modify quantitative models of simulator sickness. Such models offer a potential means for guiding the design and usage of simulator or VE systems for training and other applications. O man's O bserver Theory M odel (1982) is arguably one of the most theoretically elegant and mathematically rigorous motionsickness models in the field. Yet, although many will say that the effects of exposure duration on sickness symptomatology are obvious, it is interesting to note that O man's 1982 mathematical formulation of the O bserver Theory M odel failed to incorporate a temporal parameter or vector to account for exposure duration. In fact, increased response sensitivity was represented in this model as a consequence of a time-invariant power-law of nausea magnitude estimation. O man obviously recognizes the importance of exposure duration, as evidenced by the statement, "V irtually everyone is susceptible (to motion sickness) to some degree, provided the stimulus is appropriate and lasts long enough" (O man, 1993, p. 363). In fact, a revised version of the model (O man, 1993) added a rectification module that considers the effects of prolonged stimulation. Based on the results of the current study, a more quantitative treatment could consider integrating an empirically derived multiplicative or additive exposure duration parameter into the model that intensifies the sensory-conflict signal that produces sickness.

The results of the analysis presented here indicate that the decline in sickness with number of flights appears to be more dramatic than the increase in sickness seen with a longer exposure duration during a single flight, both in terms of the amount of change, as well as the size of the $F$ ratios. This should not necessarily be taken to indicate that adaptation to sickness over repeated flights is more important or productive than the cumulative effects of duration within a flight for several reasons. First, the data set upon which the flight duration results are based was much noisier than the data set for sickness across repeated flights. In the former, a number of simulators were used, as well as multiple scenarios for a given simulator. In addition, different types of training required different hop lengths (for example, compare take-off and landing practice to search-and-rescue flight patterns). In 
contrast, all the scenarios for the multiple-flight data set were presumably the same and were on the same simulator. Secondly, the design - and therefore the data analysis- of a repeated-measures data set is almost al ways more powerful than that of an independent group's oneway analysis, owing to the correlation across repeated measures and using each trainee as his/ her own control. If duration exposure were measured more systematically (that is, experimentally rather than observationally under comparable conditions in a similar design), the cumulative sickness increase as a result of exposure duration may be comparable to the sickness decrease seen over repeated exposures. Some evidence even indicates that the decay over time or with repeated exposures is more gradual than the adaptation rate during exposure (Welch, 1978). Further research is indicated.

As for the generalizability of these findings, it should be noted that these data are based on active-duty military pilots, a specialized group who can be expected to be relatively more resistant to unusual motion, in general, as well as more taciturn in their self-reports of illness. Therefore, it is not unreasonable to expect that, for the same stimulus, an average lay user would have stronger reactions. As for the kinematics, helicopter flight simulators generate more-dynamic scene content than do fixed-wing aircraft, if the edge rates are calculated at eye height (O wen et al., 1981; Wolpert, O wen, \& Warren, 1983), and thus should represent a particularly provocative source for the evocation and evaluation of sickness. In comparison to such flight simulators, driving simulators in downtown city street areas, with scene detail from buildings, requiring drivers to make 90 deg. turns, may have stronger kinematics than most highspeed, nap-of-the-earth helicopter flying (Kennedy \& Fowlkes, 1990). Considering all of the above factors, the application of the flight simulation results reported herein to automobile driving simulators should be direct and robust and at least should not overestimate the problem. Comparison to other simulators or VE systems should consider these same factors (that is, the characteristics of the general population of users and kinematics of the visual scene) prior to applying the results reported in this study.

\section{Conclusions}

It is clear that exposure duration and repeated exposures are critical factors in simulator-sickness outcomes (severity and incidence). M anagers of amusement parks using highly provocative VE stimuli already seem aware of cumulative duration effects, as they maintain entertainment sessions to 20 to $30 \mathrm{~min}$. at the longest, and under $3 \mathrm{~min}$. is not uncommon. M ore-systematic research is required for the design of exposure duration and repeated exposures regimens that minimize the deleterious aftereffects of VE exposure.

\section{Acknowledgements}

This material is based upon work supported in part by the $\mathrm{Na}$ tional Aeronautics and Space Administration (NASA) under grants N o. NAS9-19482 and NAS9-19453, the N ational Science Foundation (N SF) under grants N o. D M 19561266 and IRI-9624968, and the O ffice of $N$ aval Research (ONR) under grant N o. N 000149810642. Any opinions, findings, and conclusions or recommendations expressed in this material are those of the authors and do not necessarily reflect the views or the endorsement of the NASA, NSF, or ONR.

\section{References}

Biocca, F. (1992). Will simulator sickness slow down the diffusion of virtual environment technology? Presence: Teleoperatorsand Virtual Environments, 1(3), 334-343.

British Standards I nstitute. (1986). Guideto Evaluation of $\mathrm{H} \mathrm{u}$ man Exposureto Whole-Body Vertical Vibration. (BSI Document 86/ 71027). British Standard Institution.

Crampton, G. H . (Ed.) (1990). M otion and Space Sickness Boca Raton, FL: CRC Press.

Gower, D. W., Lilienthal, M. G., Kennedy, R. S., \& Fowlkes, J. E. (1987). Simulator sickness in U .S. Army and N avy fixed- and rotary-wing flight simulators. In Proceedings of the A GA R D M edical Panel Symposium in M otion Cuesin Flight Simulation and Simulator I nduced Sickness (pp. 8-1-8-20). Brussels, B elgium: N orth A tlantic Treaty O rganization, Advisory Group for Aerospace R esearch and D evelopment. 
Griffin, M . J . (1986). Effect of vibration with respect to human response. (SAE Tech Paper Series N o. 860047). Warrendale, PA: Society of Automotive Engineers.

Guignard, J. C., \& M cC auley, M . E . (1982). M otion sickness induced by complex periodic waveforms. A erospace $M$ edicine, 53, 554.

- - - (1990). The accelerative stimulus for motion sickness. In G. H. Crampton (Ed.), M otion and SpaceSickness (pp. 123-152). Boca Raton, FL: CRC Press.

H ettinger, L. J., Berbaum, K. S., Kennedy, R. S., D unlap, W. P., \& N olan, M . D . (1990). Vection and simulator sickness. Military P sychology, 2(3), 171-181.

H ettinger, L. J ., \& Riccio, G. E. (1992). Visually induced motion sickness in virtual environments. Presence: Tel eoperators and Virtual Environments, 1(3), 306-310.

Lampton, D. R., Kolasinski, E. M ., Knerr, B. W., Bliss, J. P., Bailey, J. H ., \& Witmer, B. G . (1994). Side effects and aftereffects of immersion in virtual environments. In Proceedings of the H uman Factors Soci ety 38th A nnual M eeting ( $p p$. 1154-1157). Santa M onica, CA: H uman Factors Society.

Kennedy, R. S., Berbaum, K. S., \& D rexler, J. (1994). M ethodological and measurement issues for identification of engineering features contributing to virtual reality sickness. $\mathrm{Pa}$ per presented at the I mage VII C onference, Tucson, AZ.

Kennedy, R. S., \& Fowlkes, J. E. (1990). Simulator sickness is polygenic and polysymptomatic: I mplications for research. In Proceedings of I mage V C onference (pp. 45-59). Tempe, AZ: I mage Society.

Kennedy, R. S., Jones, M. B., Lilienthal, M. G., \& H arm, D . L. (1993). Profile analysis of after-effects experienced during exposure to several virtual reality environments. In Proceedings of the 76th A er ospace M edical Panel M eeting on Virtual I nter faces: R esearch and A pplications (A GARD) (pp. 2-1-2-9). Lisbon, Portugal: A erospace M edical Association.

Kennedy, R. S., Jones, M. B., Stanney, K. M ., Ritter, A. D ., \& D rexler, J. M . (1996). H uman factors safety testing for virtual environment mission-operation training (Final Report, Contract N o. N AS9-19482). H ouston, TX: NASA Johnson Space C enter.

Kennedy, R. S., Lane, N . E., Lilienthal, M. G., Berbaum, K. S., \& H ettinger, L. J . (1992). Profile analysis of simulator sickness symptoms: Applications to virtual reality systems. Pres ence: $T \&$ VE, 1(3), 295-301.

Kennedy, R. S., Lane, N. E., Berbaum, K. S., \& Lilienthal, M . G. (1993). Profile analysis of simulator sickness symp- toms: Application to virtual environment systems. Presence: T \& VE, 1(3), 295-301.

Kennedy, R. S., \& Lilienthal, M. G. (1995). I mplications of balance disturbances following exposure to virtual reality systems. In Proceedings of the Virtual R eality A nnual I nternational Symposium '95 (pp. 35-39). Research Triangle Park, N C : IEEE Press

Kennedy, R. S., Stanney, K. M., \& J ones, M. B. (1996). Simulator sickness questionnaire: N ormative data and exposure guidelines. Presented at the 1996 A nnual Scientific M eeting of the A erospace M edical A ssociation. Atlanta, GA.

Kennedy, R. S., Tolhurst, G. C., \& Graybiel, A. (1965). The effects of visual deprivation on adaptation to a rotating environment (Tech Report N SAM 918). Pensacola, FL: N aval School of A viation M edicine.

Kolasinski, G . (1995). Simulator sicknessin virtual environments. (Tech Report 1027). O rlando: U nited States Army Research I nstitute for the Behavioral and Social Sciences.

Lampton, D. R., Kolasinski, E. M ., Knerr, B. W., Bliss, J. P., Bailey, J . H ., \& Witmer, B. G . (1994). Side effects and aftereffects of immersion in virtual environments. In Proceedings of the H uman Factors Society 38th A nnual M eeting ( $p p$. 1154-1157). Santa M onica, CA: H uman Factors Society.

Lane, N . E., \& Kennedy, R. S. (1988). A new method for quantifying sickness. D evelopment and application of the Si mulator Si ckness Questionnaire (SSQ) (E ssex O rlando Tech. Report N o. 87-3). O rlando, FL: E ssex Corporation.

M cCauley, M . E., \& Sharkey, T. J. (1992). Cybersickness: Perception of self-motion in virtual environments. Presence: $T$ \& VE, 1(3), 311-318.

M iller, E . F., \& Graybiel, A. (1970a). M otion sickness produced by head movement as a function of rotational velocity. A erospace M edicine, 41, 1180-1184.

- - - . (1970b). A provocative test for grading susceptibility to motion sickness yielding a single numerical score. A cta O to Laryngologica, Supplementum, 274. Stockholm, Sweden: Almqvist \& Wiksell Periodical Company.

O man, C. M . (1982). A heuristic mathematical model for the dynamics of sensory conflict and motion sickness. A cta Oto Laryngologica, Supplementum, 392. Stockholm, Sweden: Almqvist \& Wiksell Periodical Company.

- - - . (1993). Sensory conflict in motion sickness: An O bserver theory approach. In S. Ellis (Ed.), Pictorial communications of virtual and real environments( 2 nd ed.) (pp. 362376). London: Taylor \& Francis.

O wen, D. H ., Warren, R., Jensen, R. S., M angold, S. J., \& 
H ettinger, L. J. (1981). O ptical information for detecting loss in one's own forward speed. A cta Psychologica, 48, 203213.

Pausch, R., Crea, T., \& Conway, M . (1992). A literature survey for virtual environments: $M$ ilitary flight simulator visual systems and simulator sickness. Presence T \& VE, 1(3), 344363.

Reason, J. T. (1970). M otion sickness: A special case of sensory rearrangement. A dvancement in Science, 26, 386-393.

Reason, J. T., \& Graybiel, A. (1970). Progressive adaptation to Coriolis accelerations associated with $1 \mathrm{rpm}$ increments in the velocity of the Slow Rotation Room. A erospace M edicine, 41, 73-79.

Regan, C. (1995). An investigation into nausea and other sideeffects of head-coupled immersive virtual reality. Virtual Reality, 1(1), 17-32.

Welch, R. B. (1978). Perceptual modification: A dapting to altered sen sor y en vi ronments. N ew York: Academic Press.

Wolpert, L., O wen, D. H ., \& Warren, R. (1983). Eyeheightscaled versus ground-texture-unit-scaled metrics for the detection of loss in altitude. In Proceedings of the 2nd Symposum of A viation Psychology (pp. 513-521). 